\title{
Targeted metabolomics reveals the therapeutic impact of Eclipta prostrata on diet-induced non-alcoholic fatty liver disease in rats
}

\author{
Azza S. Helmy ${ }^{1}$, Naglaa M. Sherif ${ }^{1}$, Hassan Z. Ghanem² ${ }^{2}$ Nabaweya A. Ibrahim ${ }^{3}$, Abdel Nasser G. El Gendy ${ }^{4}$, Noha S. Hussein ${ }^{2 *}$, \\ Abdel-Hamid Z. Abdel-Hamid ${ }^{2}$ \\ ${ }^{1}$ Biochemistry Department, Faculty of Sciences, Ain-Shams University, Cairo, Egypt. \\ ${ }^{2}$ Department of Therapeutic Chemistry, National Research Centre, Cairo, Egypt. \\ ${ }^{3}$ Pharmacognosy Department, National Research Centre, Cairo, Egypt. \\ ${ }^{4}$ Medicinal and Aromatic Plants Department, National Research Centre, Cairo, Egypt.
}

\begin{tabular}{l}
\hline ARTICLE INFO \\
\hline Received on: 06/09/2018 \\
Accepted on: 15/10/2018 \\
Available online: 07/03/2019 \\
\\
\hline Key words: \\
Eclipta prostrata, GC-MS, \\
Lipidomics, NAFLD.
\end{tabular}

\begin{abstract}
Non-alcoholic fatty liver disease (NAFLD) is a growing clinical problem worldwide. The current study aimed to evaluate the therapeutic effect of the methanol extract of Eclipta prostrata (E. prostrata) in rats using gas chromatography-mass spectrometry (GC-MS) followed by multivariate and univariate data analysis. Phytochemical study of E. prostrata extract was performed using chemical, colorimetric, and high-performance liquid chromatography (HPLC) analysis. Forty-two Wistar albino rats divided into seven groups (six in each) were used in this study. Fatty liver was induced in rats using a high-fat diet together with cholesterol and cholic acid, then they were treated with different doses of E. prostrata. The histopathological examination of liver and NAFLD activity score showed significant markers for the diagnosis of the early stage of the disease. Treatment with high doses of E. prostrata (300 and $200 \mathrm{mg} / \mathrm{kg} \mathrm{BW}$ ) extract exhibited significant improvement in liver function and lipid profile in rats bearing NAFLD. Lipidomic analysis showed a discrete variability in

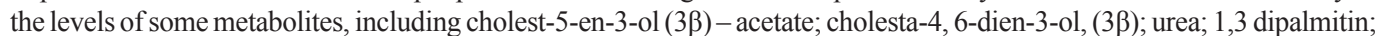
glycerol; linoleic acid; and arachidonic acid in sera of rats bearing NAFLD in contrast to healthy ones. Treatment with E. prostrata extract showed noticeable amelioration in the levels of these metabolites. The present results concluded that E. prostrata in high doses (300 and $200 \mathrm{mg} / \mathrm{kg} \mathrm{BW}$ ) had the potential efficacy to ameliorate NAFLD. Nevertheless, lipidomics could be used for the early detection of NAFLD and in the assessment of the mechanism of herbal medications.
\end{abstract}

\section{INTRODUCTION}

Approximately $35 \%$ of the world population is affected by fatty liver disease (Haga et al., 2015). The progression of non-alcoholic fatty liver disease (NAFLD) begins with steatosis, steatohepatitis, fibrosis, and finally cirrhosis (Bellentani et al., 1994). Numerous habits cause an accumulation of fats on the liver, such as excess consumption of fats daily and/or increased lipolysis in adipose tissue under insulin-resistant conditions. This leads to the higher availability of circulating non-esterified fatty acids, inappropriate synthesis and export of very low-density lipoproteins,

*Corresponding Author

Noha S. Hussein, Department of Therapeutic Chemistry, National

Research Centre, Cairo, Egypt. E-mail: dr_nohasaid @ yahoo.com

This article was presented at the 5th Euro-Mediterranean Conference and Expo on Life Sciences, Pharma and Biomedicine (BioNat-V), Limassol, Cyprus. and increased de novo fatty acid synthesis (Diraison et al., 2003). Advanced diagnostic approach to avoid the requirements for invasive procedures is demanded for novel management strategies for the growing epidemic NAFLD (Gorden et al., 2015).

Lipidomics produces large-scale lipid classes with distinctive molecular species in a biological sample (Puri et al., 2007). Lipidome analysis was used to identify lipid classes and allocation of fatty acids within these classes in the liver (Wolf and Quinn, 2008). Zehethofer and Pinto (2008) found the significance of lipidome in the pathogenesis of cancer. Plasma lipids metabolites were conducted in NAFLD and the findings showed a significant increase in total plasma monounsaturated fatty acids driven by palmitoleic (16:1 n7) and oleic (18:1 n9) acids (Puri et al., 2009).

Recently, the use of the herbal natural product as therapeutic agents has gained more interest in the world (Mushtaq et al., 2018). Among those herbs is E. prostrata (Asteraceae) 
which has high medical value (Fakurazi et al., 2008; Sun et al., 2010). Many chemical constituents such as thiophenes, coumarins, triterpenoid, saponins, steroids, and flavonoids have been isolated from E. prostrata (Kumari et al., 2006; Yuan et al., 2009). As the liver is the main organ in the synthesis and metabolism of lipid, E. prostrata can act on the liver by promoting the metabolism and accelerating the excretion of excess lipids leading to the hypolipidemic condition (Prabu et al., 2011).

So, the present study applied a lipidomic approach to detect a reproducible signature of lipid metabolites for early detection and characterization of NAFLD. In addition, to investigate the possible mechanisms of $E$. prostrata administration in improving NAFLD via amelioration of certain metabolites in comparison with a well-known lipid-lowering agent $\left(\right.$ Lipanthyl $\left.{ }^{\circledR}\right)$.

\section{MATERIALS AND METHODS}

\section{Ethics and permissions}

The procedures of the study protocol were ethically approved by Ethics Review Committee of the National Research Centre in Cairo, Egypt, which provided that the animals will not suffer at any stage of the experiment (Approval no: 13002) according to ARRIVE guidelines (Kilkenny et al., 2010).

\section{Biological materials}

Eclipta Prostrata (aerial part) was purchased from Horticultural Research Institute, Agriculture Museum. The voucher specimen was deposited in the Herbarium of National Research Centre, Cairo, Egypt under No. 997.

Experimental animals: Adult male Wistar albino rats (42) aged 5-6 weeks and weighing 160-180 g were obtained from the Animal House at National Research Centre, Egypt. The experimental animals were housed in wire cages and were maintained under standard conditions (temperature, $22^{\circ} \mathrm{C} \pm 5^{\circ} \mathrm{C}$, humidity, $55 \% \pm 5 \%$, and a 12 hour light/dark cycle). The animals had access to standard laboratory feed and water ad libitum for 7 days as an acclimatization period before starting the experiments.

\section{Chemicals}

N-methyl-N-(trimethylsilyl) trifluoroacetamide (MSTFA) with $1 \%(\mathrm{vol} / \mathrm{vol})$ trimethylchlorosilane (TMS), Methyl tert-butyl ether (MTBE), Methanol (HPLC), n-alkane (C8-C40) standard, and docosanol (an internal standard for relative quantification using GC/MS) were purchased from Sigma-Aldrich (St. Louis, MO). Cholesterol powder was purchased from Bio Basic Canada Inc. and cholic acid was purchased from Loba Chemie. All commercially available assay kits for the determination of serum liver function [aspartate aminotransferase (AST) and alanine aminotransferase (ALT)] and lipid profile were purchased from Bio Med (Cairo, Egypt). Standard diet was prepared according to the American Institute of Nutrition (Reeves et al., 1993). Thirteen standards including gallic acid, catechin, caffeic acid, syringic acid, rutin, coumaric acid, vanillin, ferulic acid, naringenin, quercetin, cinnamic acid, propyl gallate, and dihydroxy isoflavone were purchased from Sigma, Aldrich (UK) to be used as flavonoids and phenolic authentic reference.

\section{Experimental design}

Preparation and phytochemical study of E. prostrata methanol extract

Dry E. prostrata aerial part $(2 \mathrm{~kg})$ was crushed and immersed in $70 \%$ aqueous methanol solution (6 1) for 15 days. The insoluble mass was filtered out and soluble extract was concentrated with a vacuum rotary evaporator (HeidolphHei-VAP, Germany).

Phytochemical study of the dry methanol extract was performed. The triterpenes content was determined using the vanillin-perchloric acid colorimetric method (Mroczek et al., 2012). For tannins, ferric chloride reagent was used (Banso and Adeyemo, 2006) and alkaloids were measured using Mayer's reagent and Iodine/KI reagent (Abdullahi, 2013; Joshi et al., 2013). Molisch's reagent was used for carbohydrates and glycosides determinations (Joshi et al., 2013). Total phenolic and flavonoids contents were estimated according to Kim et al. (2003); Singleton and Rossi (1965), respectively. For the quantitative determination of certain flavonoids and polyphenols, HPLC analysis was carried out using an Agilent 1260 series. The separation was carried out using a $\mathrm{C}_{18}$ column $(4.6 \mathrm{~mm} \times 250 \mathrm{~mm}$ id, $5 \mu \mathrm{m})$. The mobile phase consisted of water (A) and $0.02 \%$ trifluoroacetic acid in acetonitrile (B) at a flow rate of $1 \mathrm{ml} /$ minute. The mobile phase was programmed consecutively in a linear gradient as follows: 0 minute $(80 \% \mathrm{~A}) ; 0-5$ minutes $(80 \% \mathrm{~A}) ; 5-8$ minutes $(40 \%$ A); 8-12 minutes (50\% A); $12-14$ minutes (80\% A); and $14-16$ minutes $(80 \% \mathrm{~A})$. The multi-wavelength detector was monitored at $280 \mathrm{~nm}$. The injection volume was $10 \mu \mathrm{l}$ for each of the sample solutions. The column temperature was maintained at $35^{\circ} \mathrm{C}$.

Also, Wedelolactone, the major coumarin in the dry methanolic extract of E. prostrata, was analyzed using HPLC. The standard control was dissolved in HPLC-grade methanol. The chromatographic analysis was performed at room temperature with a Cosmosil 5 C18-MS-II $(250 \mathrm{~mm} \times 4.6 \mathrm{~mm})$ column using $60 \%$ methanol as the eluent with a flow rate of $0.9 \mathrm{ml} /$ minute as described by Zhao et al. (2015).

\section{Therapeutic effect of E. prostrata}

Forty-two rats were subsequently divided into three main groups as follows:

- Negative control group: Six healthy rats received standard chow (SC) and tap water ad libitum.

- Positive control group: Six normal rats were fed a high-fat diet containing SC supplemented with cholesterol (1\%), cholic acid $(0.2 \%)$, and lard (10\%) for 8 weeks as described by Ney et al. (1988).

- Treated group: Thirty normal rats were fed a high-fat diet containing SC supplemented with cholesterol, cholic acid, and lard for 8 weeks. Rats in this group were divided into five subgroups (six in each) to be treated orally by a gavage for extra 4 weeks, with methanol extract of $E$. prostrata after dissolving in distal water at doses $300,200,100$, and $50 \mathrm{mg} / \mathrm{kg}$ body weight as described by Dhandapani (2007); and lipanthyl ${ }^{\circledR}$ drug $300 \mathrm{mg}$ at a dose $5.35 \mathrm{mg} / 200 \mathrm{~g} \mathrm{BW}$ rat (Bhardwaj and Gupta, 2012), respectively. 
At the end of the experimental period, blood samples were collected from the eyes of all rats. Samples were placed in sterilized tubes for serum separation by centrifugation at 5,000 rpm for 15 minutes. The obtained sera samples were then stored at $-80^{\circ} \mathrm{C}$ for biochemical and lipidomic analysis. Animals were then decapitated and livers were removed for histopathological examination.

\section{Biochemical analyses}

Liver function enzymes (AST and ALT) were analyzed according to Bergmeyer et al. (1976) and lipid profile [total cholesterol (TC), triacylglycerides (TAGs) and high-density lipoprotein cholesterol (HDL-CH)] were analyzed in sera samples as described by Castelli et al. (1977); Fossati and Prencipe (1982); Reitman and Frankel (1957), respectively, while low-density lipoprotein cholesterol (LDL-CH) was calculated according to Friedewald et al. (1972).

\section{Histopathological examination}

Left lobe liver sections from all rats in different groups were isolated and their preparation were carried out as demonstrated by Smith and Bruton (1978) for histopathological examination to confirm the induction of NAFLD in rats and the therapeutic effect of the methanol extract of E. prostrata in comparison with lipanthyl. Liver sections were rapidly washed with ice-cooled saline, then placed in $10 \%$ formalin saline for 24 hours, and processed routinely for embedding in paraffin. Sections of $4 \mu \mathrm{m}$ were stained with Hematoxylin \& Eosin (H\&E) and Masson's Trichrome. These sections were examined under light microscope followed by the calculation of NAFLD activity score (NAS) based on the individual scores of steatosis ( $0-3$ points), lobular inflammation ( $0-3$ points), and hepatocyte ballooning (0-2 points) to assess the severity of NAFLD (Straub and Schirmacher, 2010). A score of $<4$ was considered as steatosis

\section{Lipidome analysis using GC-MS}

\section{Serum lipidome}

Lipid was extracted from sera samples according to the method described by Matyash et al. (2008). In brief, 200 $\mu 1$ of serum from studied groups or quality control sample (prepared by equal volumes of sera from all studied groups) mixed with $1.5 \mathrm{ml}$ of methanol (98\%) containing $5 \mu$ l docosanol ( $1 \mathrm{mg} / \mathrm{ml}$, internal standard) and vortex for 5 minutes. Samples were centrifuged for 10 minutes at $5,000 \mathrm{rpm}$ and $4{ }^{\circ} \mathrm{C}$, then $5 \mathrm{ml}$ of MTBE was added to each tube, and samples were shaken for 1 hour at room temperature. Subsequently, 1.25 $\mathrm{ml}$ of MS-grade $\mathrm{H}_{2} \mathrm{O}$ was added to each tube, mixed, and allowed to stand for 10 minutes at room temperature, then centrifugation was done at $5,000 \mathrm{rpm}$ for 10 minutes to separate two phases. The upper phase was transferred into a separate tube and dried by nitrogen gas. For lipid metabolites derivatization, a volume of $100 \mu \mathrm{l}$ MSTFA containing 1\% TMS was added to the dried lipid pellets and incubated at $70^{\circ} \mathrm{C}$ for 30 minutes (Ammar et al., 2017). Agilent Technologies (GCMS system) was equipped with a gas chromatograph (7890B) and mass spectrometer detector (5977A) with HP-5MS column
(30 $\mathrm{m} \times 0.25 \mathrm{~mm}$ internal diameter and $0.25 \mu \mathrm{m}$ film thickness) at Central Laboratories Network, National Research Centre, Cairo, Egypt. The carrier gas was helium which was used to perform analyses at a flow rate of $1.0 \mathrm{ml} /$ minute, the injection volume was $1 \mu 1$ at a splitless mode with temperature program: $80^{\circ} \mathrm{C}$ for 2 minutes; rising at $5^{\circ} \mathrm{C} /$ minutes to $300^{\circ} \mathrm{C}$ and held for 5 minutes. The injector and detector were held at $280^{\circ} \mathrm{C}$ and $300^{\circ} \mathrm{C}$, respectively. Mass spectra were obtained by electron ionization (EI) at $70 \mathrm{eV}$, using a spectral range of $25-550 \mathrm{~m} / \mathrm{z}$ and solvent delay time 3.7 minutes.

For metabolites identification: Deconvolution of the detected mass spectra was performed using automated mass spectral deconvolution and identification system (AMDIS 2.64) software and national institute of standards and technology (NIST) library for identification of measured peaks via measuring the matching against reference compounds already registered. Standard fatty acids mixture (Sigma, Aldrich) was injected for confirming peak assignments. Retention indices (RI) were calculated relative to n-alkanes $\left(\mathrm{C}_{8}-\mathrm{C}_{40}\right)$ standards.

\section{Data processing for multivariate analysis}

The multivariate analysis for identified metabolites was performed using MetaboAnalyst - a comprehensive tool suite for metabolic data analysis - online software (http://www. metaboanalyst.ca) (Xia et al., 2009). Metabolites MS signals were normalized using the reference sample quality control. Data transformation is done using log transformation, especially "Pareto" scaling option prior to multivariate data analysis. After that, both unsupervised principal component analysis (PCA) and supervised partial least square-discriminant analysis (PLS-DA) were performed using this program.

\section{Statistical analysis}

Biochemical data analysis was performed via GraphPad Prism ver. 6 using the one-way analysis of variance (ANOVA). Nonparametric (Mann-Whitney test) and Kruskal-Wallis test via SPSS version 17 were used for analysis of lipidomic metabolites.

\section{RESULTS}

\section{Phytochemical study of $E$. prostrata}

The extract yield was $28.5 \%(\mathrm{w} / \mathrm{w})$ in terms of dry starting material and the phytochemical study revealed that the air dried powdered of E. prostrata (aerial part) is rich in glycosides, triterpenes, tannins, alkaloids, and coumarins. Also, its total content of polyphenols and flavonoids was $187.75 \mathrm{mg} / \mathrm{g}$ gallic extract and $144.04 \mathrm{mg} / \mathrm{g}$ rutin extract, respectively.

Data analysis by Agilent software showed the constituents of the extract as listed in Table 1. The major flavonoids were rutin and quercetin which represented 4.78 and $1.93 \mathrm{mg} / \mathrm{g}$ dry weight, respectively and the major phenolic compound was a gallic acid which represented $2.12 \mathrm{mg} / \mathrm{g}$ dry weight of the total extract.

Wedelolactone is the characteristic chemical coumarins constituent of $E$. prostrata with average content $5.00 \mathrm{mg} / \mathrm{g}$ dry weight. HPLC analysis of the wedelolactone standard showed a single peak at $350 \mathrm{~nm}$ with a retention time of approximately 7 minutes, as shown in Figure 1a and b. 
Table 1. Polyphenols and flavonoids content of methanolic extract of E. prostrata using HPLC.

\begin{tabular}{|c|c|c|c|c|c|c|c|c|c|c|}
\hline & \multicolumn{4}{|c|}{ Polyphenols } & \multicolumn{6}{|c|}{ Flavonoids } \\
\hline & Gallic Acid & Coffeic Acid & Syringic Acid & Vanillin & Cinnamic Acid & Propyl Gallate & Rutin & Naringenin & Quercetin & DihydroxyisoFlavone \\
\hline R.T (minute) & 3.18 & 4.81 & 5.16 & 8.22 & 11.1 & 10.17 & 5.61 & 9.12 & 10.59 & 10.50 \\
\hline Conc. (mg/g) & 2.12 & 0.24 & 0.17 & 0.58 & 0.09 & 0.09 & 4.78 & 0.73 & 1.93 & 0.10 \\
\hline$\%$ & 64.43 & 7.3 & 5.2 & 17.63 & 2.74 & 2.74 & 63.40 & 9.68 & 25.6 & 1.33 \\
\hline
\end{tabular}

(a)

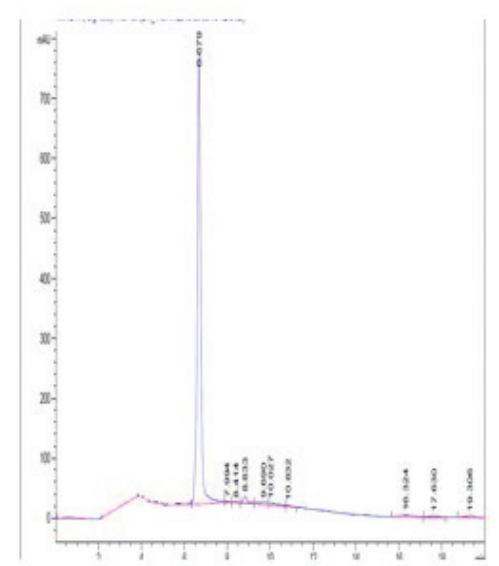

(b)

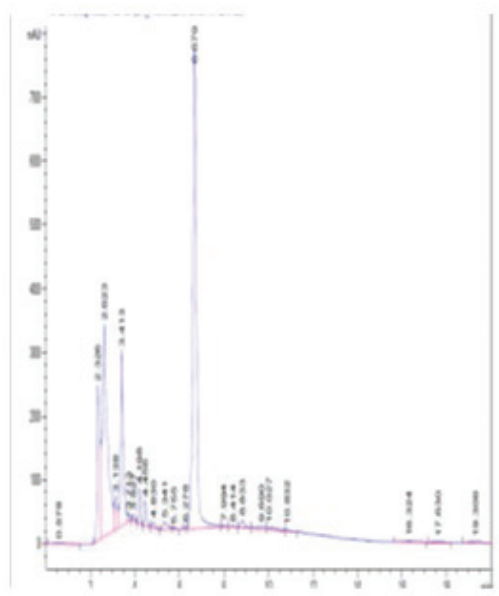

Figure 1. HPLC result of wedelolactone in standard (a) and E. Prostrata methanol extract (b).

Table 2. Biochemical analysis of blood samples of healthy, NAFLD, and treated groups.

\begin{tabular}{|c|c|c|c|c|c|c|c|c|}
\hline & & \multicolumn{2}{|c|}{ Liver function } & \multicolumn{5}{|c|}{ Lipid profile } \\
\hline \multicolumn{2}{|c|}{ Parameters Groups } & $\begin{array}{l}\text { AST } \\
(\mathbf{U} / \mathbf{l})\end{array}$ & $\begin{array}{l}\text { ALT } \\
(\mathrm{U} / \mathrm{I})\end{array}$ & $\begin{array}{c}\mathrm{TC} \\
(\mathrm{mg} / \mathrm{dl})\end{array}$ & $\begin{array}{c}\text { TAG } \\
(\mathrm{mg} / \mathrm{dl})\end{array}$ & $\begin{array}{l}\text { HDL-C } \\
(\mathrm{mg} / \mathrm{dl})\end{array}$ & $\begin{array}{l}\text { LDL-C } \\
(\mathrm{mg} / \mathrm{dl})\end{array}$ & $\begin{array}{c}\text { HDL-C/LDL-C } \\
\text { ratio }\end{array}$ \\
\hline \multicolumn{2}{|l|}{ Negative control } & $31.16^{\mathrm{a}} \pm 0.42$ & $20.20^{\mathrm{a}} \pm 0.67$ & $96.00^{\mathrm{a}} \pm 0.83$ & $62.45^{\mathrm{a}} \pm 0.93$ & $46.69^{\mathrm{a}} \pm 0.38$ & $36.82^{\mathrm{a}} \pm 1.28$ & $1.26^{\mathrm{a}} \pm 0.04$ \\
\hline \multirow{2}{*}{\multicolumn{2}{|c|}{$\begin{array}{l}\text { Positive control } \\
\text { (\%Change) }\end{array}$}} & $95.46^{\mathrm{b}} \pm 0.47$ & $43.14^{b} \pm 0.61$ & $200.18^{\mathrm{b}} \pm 1.05$ & $185.22^{\mathrm{b}} \pm 0.62$ & $23.11^{\mathrm{b}} \pm 0.48$ & $140.07^{b} \pm 0.96$ & $0.16^{b} \pm 0.003$ \\
\hline & & 206.35 & 113.54 & 108.53 & 196.59 & -50.50 & 280.4 & -87.30 \\
\hline \multirow[t]{10}{*}{ Treated Group } & Lipanthyl & $52.13^{\mathrm{c}} \pm 0.66$ & $26.28^{\circ} \pm 0.53$ & $125.11^{\mathrm{c}} \pm 0.56$ & $85.00^{c} \pm 0.53$ & $37.86^{\circ} \pm 0.49$ & $70.14^{\mathrm{c}} \pm 0.93$ & $0.54^{c} \pm 0.03$ \\
\hline & (\%Change) & 67.29 & 30.10 & 30.32 & 36.11 & -18.91 & 90.58 & -57.14 \\
\hline & E. prostrata $(300)$ & $54.02^{\mathrm{c}} \pm 0.57$ & $28.34^{\mathfrak{c}} \pm 0.46$ & $139.20^{\mathrm{d}} \pm 0.51$ & $94.19^{d} \pm 0.57$ & $33.08^{\mathrm{d}} \pm 0.46$ & $87.28^{\mathrm{d}} \pm 0.76$ & $0.37^{\mathrm{d}} \pm 0.02$ \\
\hline & & 73.36 & 40.30 & 45.00 & 50.82 & -29.15 & 137.05 & -70.63 \\
\hline & E. prostrata $(200)$ & $73.07^{\mathrm{d}} \pm 0.56$ & $32.37^{\mathrm{d}} \pm 0.83$ & $158.34^{\mathrm{e}} \pm 0.46$ & $102.38^{\mathrm{e}} \pm 0.69$ & $30.85^{\mathrm{d}} \pm 0.38$ & $107.01^{e} \pm 0.54$ & $0.29^{\mathrm{e}} \pm 0.01$ \\
\hline & & 134.50 & 60.25 & 64.94 & 63.94 & -33.93 & 190.63 & -76.98 \\
\hline & E. prostrata $(100)$ & $80.64^{\mathfrak{e}} \pm 0.39$ & $36.24^{e} \pm 0.61$ & $170.22^{\mathrm{f}} \pm 0.52$ & $128.26^{\mathrm{f}} \pm 0.56$ & $28.14^{\mathrm{e}} \pm 0.58$ & $116.21^{\mathrm{f}} \pm 0.33$ & $0.24^{\mathrm{f}} \pm 0.01$ \\
\hline & & 158.79 & 79.41 & 77.31 & 105.38 & -39.73 & 215.62 & -80.95 \\
\hline & E. prostrata $(50)$ & $88.38^{\mathrm{f}} \pm 0.54$ & $39.29^{\mathrm{r}} \pm 0.47$ & $185.70^{\mathrm{g}} \pm 0.88$ & $140.33^{\mathrm{g}} \pm 0.40$ & $26.23^{\mathrm{e}} \pm 0.27$ & $131.40^{\mathrm{g}} \pm 0.79$ & $0.20^{\mathrm{g}} \pm 0.003$ \\
\hline & & 183.63 & 94.50 & 93.44 & 124.71 & -43.82 & 256.87 & -84.13 \\
\hline
\end{tabular}

Data are represented in this table as Mean $\pm \mathrm{SE}$, different letters are significant at $p \leq 0.05$.

\section{Biochemical assay}

Toxicity assay of E. prostrata is carried out and the results reported its safety up to $600 \mathrm{mg} / \mathrm{kg}$ BW. Data listed in Table 2 showed a significant elevation in the levels of ALT, AST, TC, triacylglycerides (TAGs), and LDL-C. The results also showed depletion in the level of HDL-C and HDL/LDL-C ratio in the serum of rats bearing NAFLD. It is also noticed that liver enzymes and lipid profile were markedly ameliorated in treated animals with high and moderate doses of E. prostrata compared with the standard drug lipanthyl.

\section{Serum metabolites profiling}

A total of 35 serum metabolites including [free fatty acids (FFAs), glycerolipids, sterols, oxysterols, cholesteryl ester, and sugars], its retention times (Rt), RI, and the mass-to-charge ratio $(\mathrm{m} / \mathrm{z})$ were represented in Supplementary Table 1 . The percentage of different lipid types in normal healthy and NAFLD groups were represented in Figure 2. PCA was first applied to the GC/MS dataset with score plot (Supplementary Figure 1) showing somewhat not clear segregation of sample groups. The first two components (PC1 and PC2) explained $26.2 \%$ and $20.2 \%$ of the total 
variance, respectively. Consequently, a supervised data analysis method was adopted to derive better samples classification. PLSDA was applied. PLS-DA score plot (Fig. 3a) showed better and more clear discrimination among sample groups with $R^{2}$ value $(0.69)$ and $Q^{2}$ value $(0.56)$.

Metabolites contributing for groups segregation revealed from variable importance in projection (VIP) score plot $(>1)$ (Fig. 3b) include cholest-5-en-3-ol (3ß)- acetate, urea, cholesta4,6-dien-3-ol, (3ß)-, myristic acid 1, 1,3-dipalmitin, glycerol, palmitic acid, 2-monostearin, myristic acid, and stearic acid. The levels of these metabolites were different in all studied groups.

For further separation among groups, the Hierarchical Clustering/Dendrograms model was displayed as shown in Figure 3c. This Dendrograms model showed that fatty liver group was located far from the normal group, while treated groups were found in between and suggested that the dose $300 \mathrm{mg} / \mathrm{kg}$ had the most ameliorative effect against NAFLD after lipanthyl group. PLSDA model and its derived VIP score plot were further employed to identify metabolites markers related to NAFLD by modeling healthy control versus non-alcoholic fatty liver rats (Fig. 4a and b). The PLS-DA model exhibits $R^{2}$ value (0.98) and $Q^{2}$ value (0.96), with control and NAFLD groups being clearly discriminated from each other. Cholest-5-en-3-ol (3 $\beta$ )- acetate, cholesta-4,6-dien-3ol, (3ß)-, urea, glycerol, and 1,3-dipalmitin were most elevated in NAFLD group, whereas arachidonic acid and linoleic acid were detected at lower levels as compared to the healthy control one.

PLS-DA model encompassing the three groups: normal control, NAFL untreated, and lipanthyl drug-treated groups were attempted (Fig. 5a and b). Score plot showed discrimination between NAFL group and normal control, with lipanthyl treated animal group clustering closer to normal control. The corresponding VIP score

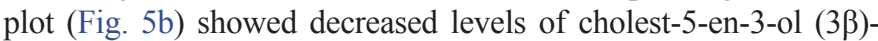
acetate, cholesta-4,6-dien-3-ol, (3ß)-, glycerol, urea, 1,3-dipalmitin, and 2-monostearin concurrent with increasing in linoleic acid levels in the serum lipanthyl drug group compared with NAFL group. On the other hand, treated groups of E. prostrata at doses 300, 200, 100 , and $50 \mathrm{mg} / \mathrm{kg} \mathrm{BW}$ were found also the ones that clustered most closely to the control group in the PLS-DA plot. Consequently, another PLS-DA plots were constructed encompassing untreated
NAFLD group, normal control with different doses of $E$. prostrata $(300,200,100,50 \mathrm{mg} / \mathrm{Kg}$ BW) separately as shown in [(Suppl. Fig.S2a), (Suppl.Fig.S3a), (Suppl.Fig.S4a) and (Suppl.Fig.S5a), respectively]. Metabolites observed from VIP score plot in treated group administered E. prostrata at dose $300 \mathrm{mg} / \mathrm{kg}$ BW model

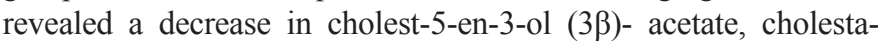
4,6-dien-3-ol, (3ß)-, glycerol, urea, 2-monostearin, and an increase in arachidonic and linoleic acids (Supplementary Figure 2b) as compared with NAFLD group. While, metabolites observed from VIP score plots in treated groups administered $E$. prostrata at doses 200,100 , and $50 \mathrm{mg} / \mathrm{kg}$ BW model revealed a decrease in cholest-

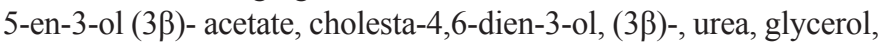
1,3-dipalmitin, 2-monostearin, and an increase in arachidonic acid and linoleic acid levels (Supplementary Figures 3b, 4b, and 5b), respectively as compared with NAFLD group. Fold change of these metabolites was performed using univariate data analysis of Metabo-Analyst (Table 3).

Also, non-parametric Kruskal-Wallis test pointed out

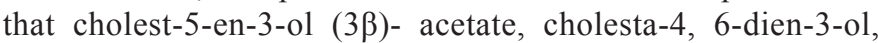
$(3 \beta)$, urea, 1, 3-dipalmitin, glycerol, linoleic acid, arachidonic acid, and cholesterol at $(p \leq 0.05)$ are the most impacted metabolites between all studied groups and suggested for discrimination between healthy and NAFLD ones. From these results, there are seven metabolites common in multivariate analysis using PLS-DA and univariate analysis using KruskalWallis test that (cholest-5-en-3-ol (3ß)- acetate, cholesta-4,6dien-3-ol, (3ß), urea, 1,3 dipalmitin, glycerol, linoleic acid and arachidonic acid). By following the PLS-DA VIP score plots derived results, fold change suggested a significant decrease $(\mathrm{P} \leq 0.05)$, using non-parametric Mann-Whitney test, in arachidonic and linoleic acids levels versus an increase in

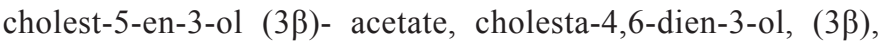
urea, 1,3 dipalmitin and glycerol amounts in NAFLD group as compared to healthy group. The group administered lipanthyl drug and all doses of E. prostrata methanol extract showed a marked increase in arachidonic and linoleic acids levels versus a decrease in cholest-5-en-3-ol (3ß)- acetate, cholesta-4,6-dien-

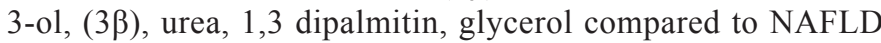
group gradually. (a)

Normal control group

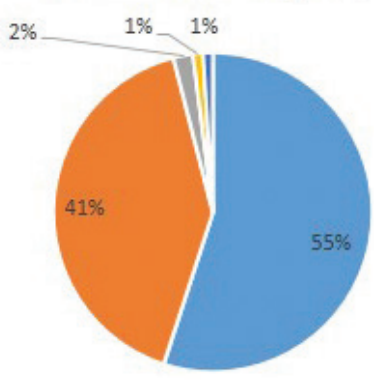

(b)
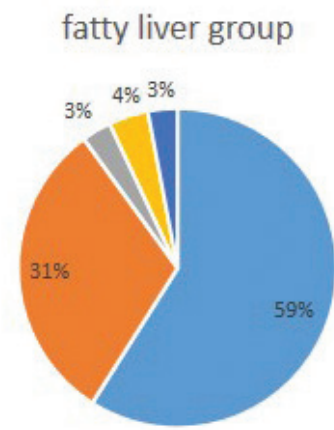

- Glycerolipids = FFA " sterols " oxysterols "CE

Figure 2. The percentages of different lipid types in normal healthy control group (a) and fatty liver group (b). FFA = free fatty acids; $\mathrm{CE}=$ cholesteryl ester. 
(a)

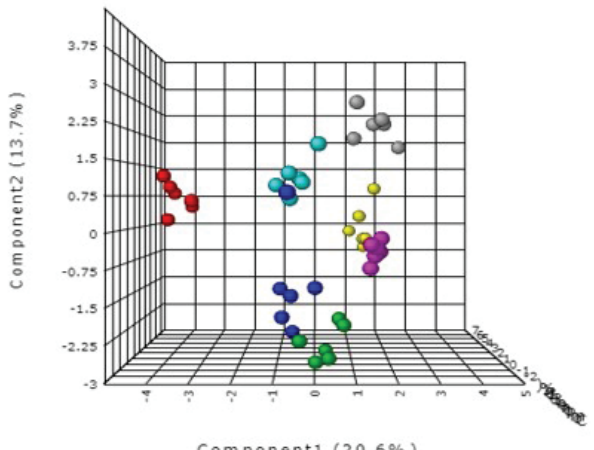

(c)

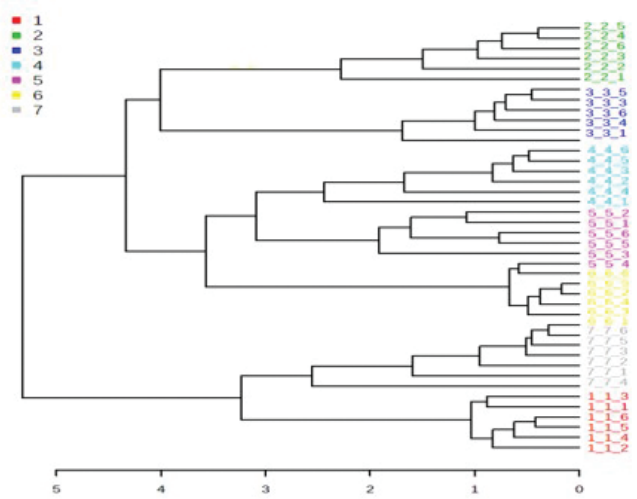

(b)

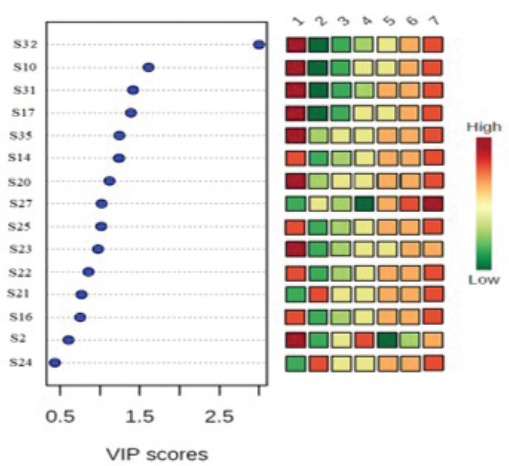

Figure 3. PLS-DA score plots obtained from modeling serum metabolites in the different groups. (a) The score plot showing a separation of healthy control animals, NAFLD rats, and treated groups. (b) VIP score plot for determination of important metabolites (VIP score > 1). S32: Cholest-5-en-3-ol (3ß)- acetate, S10: Urea, S31: Cholesta-4,6-dien-3-ol, (3ß)-, S17: Myristic acid 1, S35: 1,3-Dipalmitin, S14: Glycerol, S20:Palmitic acid, S27: 2-Monostearin, S25: Myristic acid2, S23: Stearic acid. Peaks numbering follow that listed in Supplementary Table 1 for metabolite identification using GC-Ms. The colored boxes on the right indicated the relative concentrations of the corresponding metabolite in each group under study. (c) Hierarchical Clustering/Dendrograms of the six groups with fatty liver group showing separation from the normal control group and treated groups in serum samples. Sample codes are presented as follows: (1) fatty liver group, (2) healthy control, (3) standard drug lipanthyl, and extract doses: $300 \mathrm{mg} / \mathrm{kg} \mathrm{BW} \mathrm{(4),} 200 \mathrm{mg} / \mathrm{kg} \mathrm{BW}(5), 100 \mathrm{mg} / \mathrm{kg} \mathrm{BW}(6)$, and $50 \mathrm{mg} / \mathrm{kg} \mathrm{BW}$ (7).

(a)

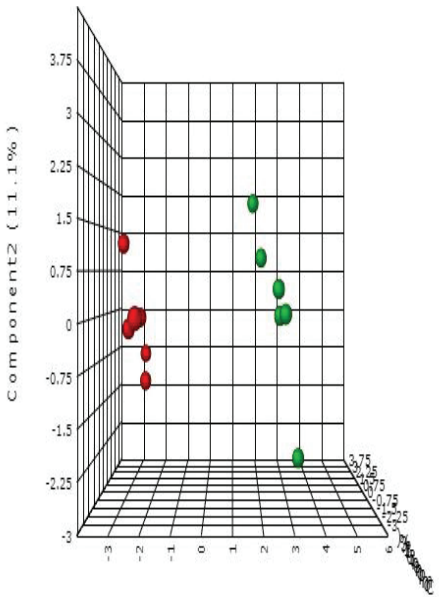

Component1 $(57.7 \%)$ (b)

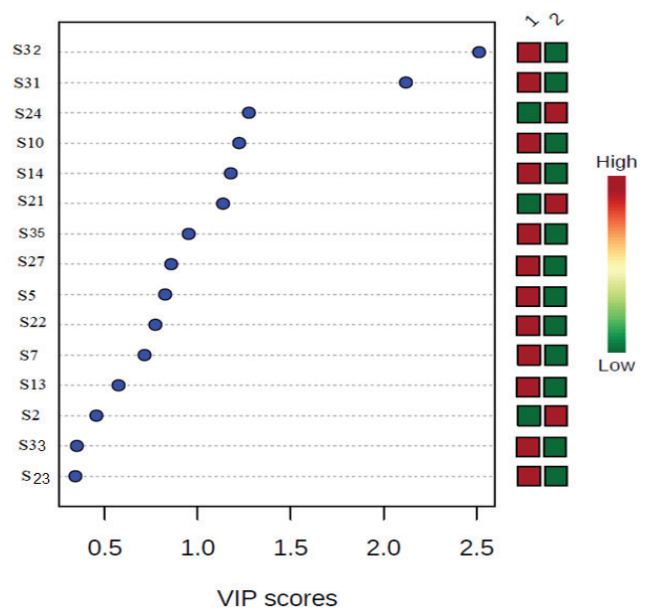

Figure 4. PLS-DA score plots obtained from GC-MS data by modeling NAFLD (1) versus healthy control groups (2) against each other. (a) Score plot of PC1 and PC2.

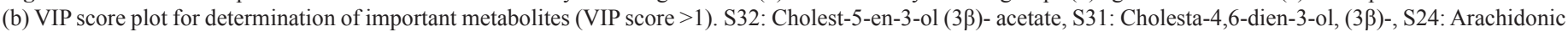
acid, S10: Urea, S14: Glycerol, S21: Linoleic acid, S35: 1,3-dipalmitin. Peaks numbering follow that listed in Supplementary Table 1 for metabolite identification using GC-Ms. The colored boxes on the right indicated the relative concentrations of the corresponding metabolite in each group under study. 


\section{Histopathology examination}

Liver sections showed distinct differences between healthy control and fatty liver groups and how these changes were gradually eliminated after the administration of the methanolic extract of E. prostrata as compared with standard drug lipanthyl. Results pointed out that the most effective dose of E. prostrata extract was $300 \mathrm{mg} / \mathrm{kg} \mathrm{BW}$. The liver sections in the control group showed hepatic tissue with preserved (intact) lobular hepatic architecture and normal morphological appearance $(\mathrm{H} \& \mathrm{E}, \times 400)$ and (Masson Trichrome, $\times 400)$. In contrast, the NAFLD group showed preserved (intact) lobular hepatic architecture, hepatocyte ballooning, severe micro and macrovesicular steatosis (black arrows), and moderate lobular inflammation $[\mathrm{H} \& \mathrm{E}, \times 400($ Masson Trichrome, $\times 400)]$ as shown in Figure $6 \mathrm{a}$ and $\mathrm{b}$, leading to about 12 -fold change $(p<0.001)$ increase in NAS. This value was significantly decreased in all treated groups as compared with NAFLD group, as shown in Figure $6 \mathrm{c}$, due to decreasing hepatocyte ballooning and micro and macrovesicular steatosis. NAS calculation is shown in Figure 6c. Masson Trichrome staining showed no evidence of fibrosis. These results reported that the current study was performed during the early stages of NAFLD where NAS equal 3.5 and it can be prevented from progression through administering $E$. prostrata methanolic extract.

\section{DISCUSSION}

In the present study, ALT and AST were measured as markers of liver injury and results showed that they could be used as surrogate indicators of NAFLD with lipid profile. These illustrations give some support to the findings of Sanyal et al. (2015) who pointed out that NAFLD was the most common cause of abnormal liver biochemistry. ALT and AST showed significant amelioration in groups treated with E. prostrata. The therapeutic activity of $E$. prostrata could be due to its active constituents (flavonoids, alkaloids, tannins, triterpenes, and coumarins) which have free radicals scavenging properties and indirectly increase the antioxidants levels by decreasing the levels of lipid peroxidative products (Arun and Balasubramanian, 2011).

The study of Bravo et al. (2011) pointed out that feeding rats with a high-fat diet induce insulin resistance, hypertriglyceridemia, hepatic steatosis, and liver damage as characteristic features of NAFLD. In our study, the enhancement in the lipid profile of treated rats with E. prostrata might be attributed to the lipid-lowering activity of this plant which is linked with the down-regulation of "hydroxy-3-methylglutaryl-CoA reductase (HMGR)," a key enzyme in cholesterol biosynthesis and the up-regulation of peroxisome proliferatoractivated receptor $\alpha$ (PPAR $\alpha)$ (Tammela et al., 2005), low density lipoprotein receptor (Arun and Balasubramanian, 2011), lecithin-cholesterol transferase (Rader et al., 2009), and scavenger receptor class B type I receptor SR-BI, which is a well-known HDL receptor (Kozarsky et al., 1997).

In this study, NAFLD was associated with perturbations in lipid metabolic pathways in terms of FFAs and elevation in glycerolipids (1,3-dipalmitin and glycerol), oxysterols [cholesta4,6-dien-3-ol, (3ß)], cholesteryl ester (cholest 5-en-3ol-3 acetate), and urea.

The present study indicated lower levels of linoleic acid (18:2 n6) and arachidonic acid (20:4 n6) in NAFLD group which may be due to their consumption for producing eicosanoids. These eicosanoids play a vital role in the progression of hepatotoxicity (Maciejewska et al., 2015; Puri et al., 2007). (a)

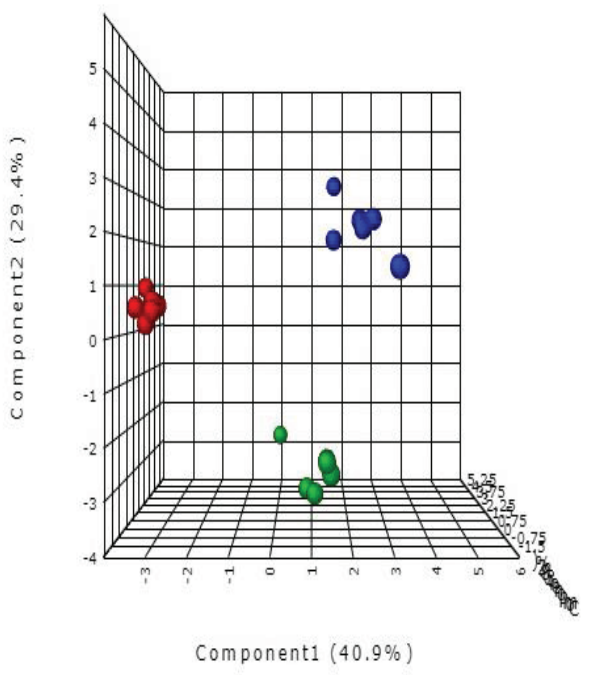

(b)

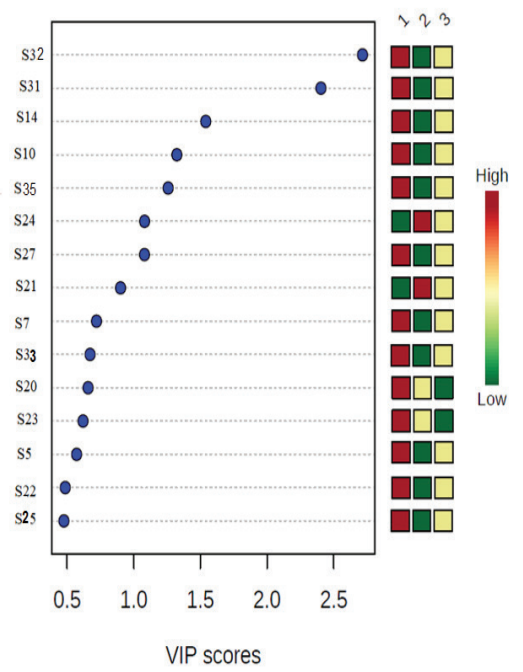

Figure 5. PLS-DA score plots obtained from modeling NAFLD (1) healthy control (2) and (3) lipanthyl drug-treated groups. (a) Score plot of PC1 and PC2. (b) VIP

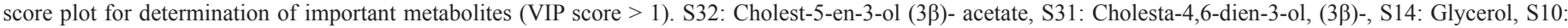
Urea, S35: 1,3-dipalmitin, S27: 2-Monostearin, S21: Linoleic acid. Peaks numbering follow that listed in Supplementary Table 1 for metabolite identification using GC-Ms. The colored boxes on the right indicate the relative concentrations of the corresponding metabolite in each group under study. 
Table 3. The common metabolite level alterations according to PLS-DA score (VIP $>1)$, Kruskal-Wallis test analysis and fold change in all treated experimental groups.

\begin{tabular}{|c|c|c|c|c|c|c|}
\hline \multirow{2}{*}{ Metabolites } & \multicolumn{6}{|c|}{ Fold change } \\
\hline & $\mathbf{F} / \mathbf{N}$ & $\mathrm{D} / \mathrm{F}$ & $300 / F$ & $200 / F$ & $100 / \mathrm{F}$ & $50 / \mathbf{F}$ \\
\hline${ }^{\mathrm{a}} \mathrm{Urea}, \mathrm{S} 10$ & $* 6.39$ & $* 0.28$ & $* 0.42$ & $* 0.53$ & $* 0.74$ & $* 0.87$ \\
\hline${ }^{\mathrm{a}}$ Glycerol, S14 & $* 4.05$ & $* 0.37$ & $* 0.50$ & $* 0.67$ & $* 0.80$ & $* 0.87$ \\
\hline${ }^{\mathrm{a}}$ Linoleic acid, S21 & $* 0.12$ & *5.43 & $* 4.51$ & $* 2.97$ & $* 1.89$ & *1.44 \\
\hline${ }^{\text {aArachidonic acid, S24 }}$ & $* 0.15$ & $* 4.18$ & *3.28 & $* 2.58$ & $* 1.80$ & *1.43 \\
\hline${ }^{\mathrm{a} C}$ Cholesta-4,6-dien-3-ol, (3ß)-, S31 & $* 4.75$ & $* 0.45$ & $* 0.53$ & $* 0.62$ & $* 0.69$ & $* 0.79$ \\
\hline${ }^{\mathrm{a} C}$ Cholest 5 -en-3ol $-3 \beta$ acetate, $\mathrm{S} 32$ & $* 6.42$ & $* 0.32$ & $* 0.47$ & $* 0.63$ & $* 0.77$ & $* 0.88$ \\
\hline a1,3-dipalmitin, S35 & $* 3.38$ & $* 0.37$ & $* 0.48$ & $* 0.58$ & $* 0.69$ & $* 0.78$ \\
\hline
\end{tabular}

Fold change was performed by using Metabo-Analyst ver. 3; the Statistical comparisons equal non-parametric Mann-Whitney test was performed. Results were considered only significant if $P \leq 0.05$. a : denotes for metabolites showing significant using Kruskal-Wallis test analysis.*: denotes for metabolites showing significant difference among treated, normal and fatty liver groups. Sample codes are presented as follows normal healthy (N), drug (D), fatty liver (F), 300, 200, 100, and 50 mg/Kg BW (different dosage of E. prostrata extract).

a
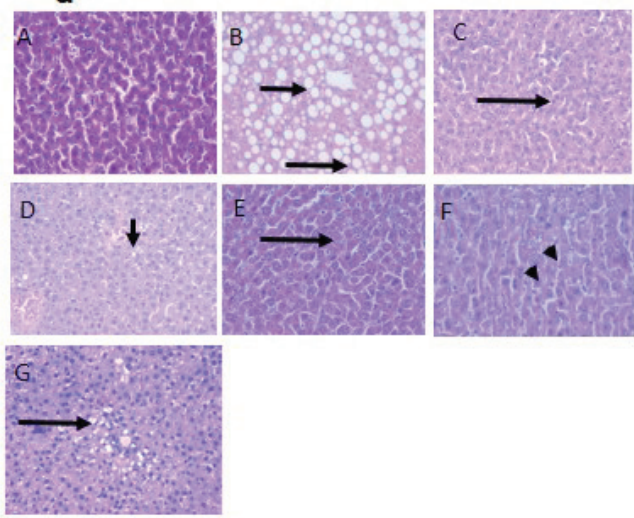

b
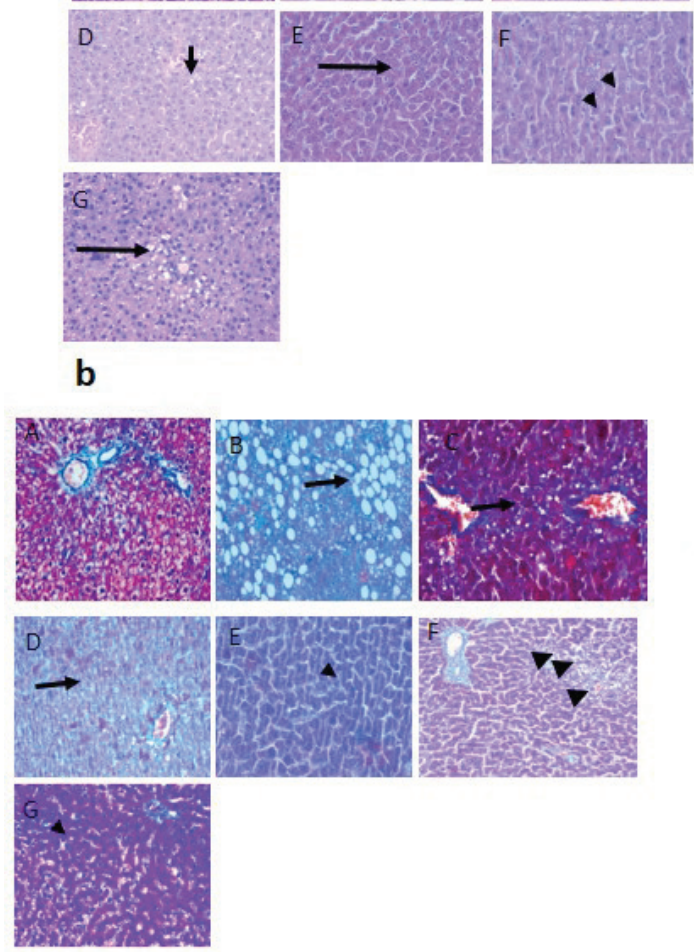

C

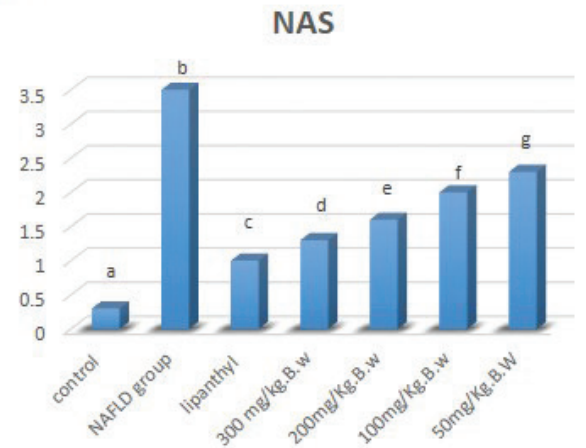

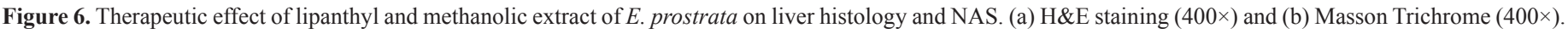

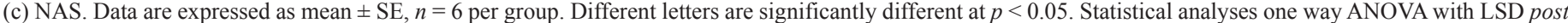

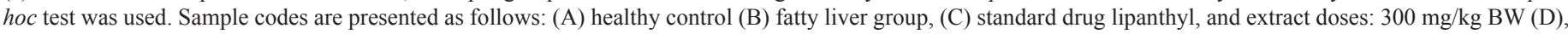
$200 \mathrm{mg} / \mathrm{kg} \mathrm{BW}(\mathrm{E}), 100 \mathrm{mg} / \mathrm{kg} \mathrm{BW}(\mathrm{F})$, and $50 \mathrm{mg} / \mathrm{kg} \mathrm{BW}(\mathrm{G})$.

Lipid metabolism abnormalities and insulin resistance in NAFLD increase the glycerolipids, as in the study, where glycerol and 1,3-dipalmitin levels were raised and sequentially accumulation of TAGs was observed which were produced through esterification of FFA with glycerol-3-phosphate (Rodriguez et al., 2014).
Interestingly, three oxysterol compounds, cholesta-4, 6-dien-3-ol, cholesta-4, 6-dien-3-one, and cholesta-3, 5-dien-7one are derived from the oxidation of cholesterol in the erythrocyte membrane by reactive oxygen species. In our study, we noticed the increased level of cholesta-4,6 dien-3-ol in the NAFLD group 
as compared with healthy control group indicating the elevated cholesterol level in this group and its oxidation products (Liu and Shan, 2006) and these results agreed with Serviddio et al. (2016) who reported that oxysterols play a vital role in the pathogenesis of NAFLD. Puri et al. (2007) reported that the observed raised cholesterol level in NAFLD was due to numerous aberrations that included deterioration of adenosine triphosphate synthesis which increases the 3-hydroxy-3-methylglutaryl coenzyme A reductase activity and mitochondrial abnormalities. In addition, cholest 5-en-3ol-3 acetate which is present in all human fluids and organs is elevated in plasma in association with different lipid-related diseases as atherosclerosis and NAFLD due to the decreased level of high-density lipoprotein (Kuivenhoven et al., 1998).

An increased urea level in NAFLD found in the present results is similar to the clinical report of Targher et al. (2011) in patients with NAFLD and higher blood urea nitrogen which could have a prognostic value.

Theameliorationinthepreviousmetaboliteslevelsobserved in $E$. prostrata extract treated animals may be due to the presence of main active constituents as flavonoids (rutin and quercetin), phenols [gallic acid (GA)], and coumarins (wedelolactone) which regulate lipid metabolism. Wu et al. (2011) reported that the flavonoid rutin can improve hepatic injury by promoting activities of some enzymes as adenosine 5-monophosphate (AMP)-activated protein kinase (AMPK), sterol regulatory element binding proteins-1, 3-hydroxy3-methylglutarylcoenzyme A (HMG-CoA) reductase, glycerol-3phosphate acyltransferase, fatty acid synthase, and acetyl-coenzyme carboxylase which are involved in lipid synthesis and metabolism. Moreover, Porras and Nistal (2017) observed the reduction in insulin resistance and NAFLD activity score that accompanied quercetin administration via its reduction of lipid accumulation is due to its modulation of lipid metabolism. Chao et al. (2014) reported that the target of GA treatment is lipid metabolism and proved that GA can improve lipid homeostasis in high-fat-diet-induced NAFLD in mice. Wedelolactone may promote lipid metabolism by upregulation of AMPK and PPAR $\alpha$ levels and thus was found to be beneficial against hepatic steatosis (Zhao et al., 2015).

\section{CONCLUSION}

The current study represented the main altered lipid metabolites related to NAFLD in serum samples using a lipidomic approach to act as key points to control this disease in the early disease stage. This study also highlights the therapeutic effect of $E$. prostrata through its regulation to the progression of NAFLD and amelioration of these metabolite profiles and pointed that the highest dose used in the present study of methanol extract of $E$. prostrata has the maximum therapeutic effect compared with lipanthyl drug.

\section{ACKNOWLEDGMENTS}

The author thanks Therapeutic Chemistry Department, National Research Centre (NRC) for providing facilities for doing this research. Academy of Scientific Research \& Technology for supporting this study.

\section{CONFLICT OF INTEREST}

The authors have no conflicts of interest to declare.

\section{FUNDING}

This research was financially supported by the National Research Centre and Academy of Scientific Research \& Technology, Cairo, Egypt.

\section{ABBREVIATIONS}

$\mathrm{ACC}$

ALT:

AMPK:

AST:

DAG:

FFA:

GC-Ms:

GPAT:

HDL-C:

HMG CoA:

HMGCR:

HPLC:

LCAT:

LDL-C:

LDLR:

MS-grade $\mathrm{H}_{2} \mathrm{O}$ :

NAFLD:

NASH:

PCA:

PLS-DA:

PPAR $\alpha$ :

SR-BI:

SREBP-1c:

SS:

TAG:

TC:

acetyl-coenzyme carboxylase acids

alanine aminotransferase

adenosine monophosphate-activated protein kinase

aspartate aminotransferase

diacylglycerol

free fatty acid

gas chromatography-mass spectrometry

glycerol-3-phosphate acyltransferase

high-density lipoprotein cholesterol

$\beta$-Hydroxy $\beta$-methylglutaryl-CoA

hydroxy-3-methyl-glutaryl-CoA reductase

high-performance liquid chromatography

lecithin-cholesterol transferase

low-density lipoprotein cholesterol

low-density lipoprotein receptor

mass spectra high-grade water

non-alcoholic fatty liver disease

non-alcoholic steatohepatitis

principal component analysis

partial least square discriminate analysis peroxisome proliferator-activated receptor scavenger receptor class B type I receptor sterol regulatory element binding protein-1c simple steatosis

triacylglycerides

total cholesterol

\section{AUTHOR CONTRIBUTIONS}

Azza Salah Helmy and Naglaa M Sherif conceived and coordinated the study. Hassan Zaki Ghanem helped in performing experiments. Nabaweya Ali Ibrahim providing an extract E. prostrata. Abdel-Nasser G El-Gendy helped in identifications of metabolites and in statistical analysis. Noha Said Hussein performed experiments and prepared the manuscript. AbdelHamid Zaki Abdel-Hamid involved in designing the hypothesis and experiments, conceived and coordinated the study and made a revision to the manuscript. All authors read and approved the final version of the manuscript.

\section{REFERENCES}

Abdullahi MN. Evaluation of phytochemical screening and analgesic activity of aqueous extract of the leaves of Microtrichia perotitii Dc (Asteraceae) in mice using hotplate method. Med Plant Res, 2013; $3: 37-43$.

Ammar NM, Farag MA, Kholeif TE, Metwally NS, El-Sheikh NM, El Gendy AN, Abdel-Hamid AH. Serum metabolomics reveals the mechanistic role of functional foods and exercise for obesity management in rats. J Pharm Biomed Anal, 2017; 142:91-101.

Arun K, Balasubramanian U. Comparative study on Hepatoprotective activity of Aegle marmelos and Eclipta alba against alcohol induced in albino rats. Int J Environ Sci, 2011; 2:401-14. 
Banso A, Adeyemo S. Phytochemical screening and antimicrobial assessment of Abutilon mauritianum, Bacopa monnifera and Datura stramonium. Biokemistri, 2006; 18:39-44.

Bellentani S, Tiribelli C, Saccoccio G, Sodde M, Fratti N, De Martin C, Christianini G. Prevalence of chronic liver disease in the general population of northern Italy: the Dionysos Study. Hepatology, 1994; 20:1442-9.

Bergmeyer HU, Bowers GNJ, Horder M, Moss DW. Provisional recommendations on IFCC methods for the measurement of catalytic concentrations of enzymes. Part 2. IFCC method for aspartate aminotransferase. Clin Chim Acta, 1976; 70:F19-29.

Bhardwaj S, Gupta D. Study of acute, Subacute and chronic toxicity test. Int J Adv Res Pharm Bio Sci, 2012; 1:103-129.

Bravo E, Palleschi S, Aspichueta P, Buqué X, Rossi B, Cano A, Napolitano M, Ochoa B, Botham KM. High fat diet-induced non alcoholic fatty liver disease in rats is associated with hyperhomocysteinemia caused by down regulation of the transsulphuration pathway. Lipids Health Dis, $2011 ; 10: 60$.

Castelli WP, Doyle JT, Gordon T, Hames CG, Hjortland MC, Hulley SB, Kagan A, Zukel WJ. HDL cholesterol and other lipids in coronary heart disease. The cooperative lipoprotein phenotyping study. Circulation, 1977; 55:767-72.

Chao J, Huo T-I, Cheng H-Y, Tsai JC, Liao JW, Lee MS, Qin XM, Hsieh MT, Pao LH, Peng WH. Gallic acid ameliorated impaired glucose and lipid homeostasis in high fat diet-induced NAFLD mice. PLoS One, 2014; 9:e96969.

Dhandapani R. Hypolipidemic activity of Eclipta prostrata (L.) L. leaf extract in atherogenic diet induced hyperlipidemic rats. Indian J Exp Biol, 2007; 45:617-9.

Diraison F, Moulin P, Beylot M. Contribution of hepatic de novo lipogenesis and reesterification of plasma non esterified fatty acids to plasma triglyceride synthesis during non-alcoholic fatty liver disease. Diabetes Metab, 2003; 29:478-85.

Fakurazi S, Hairuszah I, Nanthini U. Moringa oleifera Lam prevents acetaminophen induced liver injury through restoration of glutathione level. Food Chem Toxicol, 2008; 46:2611-5.

Fossati P, Prencipe L. Serum triglycerides determined colorimetrically with an enzyme that produces hydrogen peroxide. Clin Chem, 1982; 28:2077-80.

Friedewald WT, Levy RI, Fredrickson DS. Estimation of the concentration of low-density lipoprotein cholesterol in plasma, without use of the preparative ultracentrifuge. Clin Chem, 1972; 18:499-502.

Gorden DL, Myers DS, Ivanova PT, Fahy E, Maurya MR, Gupta S, Min J, Spann NJ, McDonald JG, Kelly SL, Duan J. Biomarkers of NAFLD progression: a lipidomics approach to an epidemic. J Lipid Res, $2015 ; 56: 722-36$

Haga Y, Kanda T, Sasaki R, Nakamura M, Nakamoto S, Yokosuka O. Nonalcoholic fatty liver disease and hepatic cirrhosis: comparison with viral hepatitis-associated steatosis. World J Gastroenterol, 2015; 21:12989-95.

Joshi A, Bhobe M, Sattarkar A. Phytochemical investigation of the roots of Grewia microcos Linn. J Chem Pharm Res, 2013; 5:80-7.

Kilkenny C, Browne WJ, Cuthill IC, Emerson M, Altman DG. Improving bioscience research reporting: the ARRIVE guidelines for reporting animal research. PLoS Biol, 2010; 8:e1000412.

Kim D-O, Chun OK, Kim YJ, Moon H-Y, Lee CY. Quantification of polyphenolics and their antioxidant capacity in fresh plums. J Agric Food Chem, 2003; 51:6509-15.

Kozarsky KF, Donahee MH, Rigotti A, Iqbal SN, Edelman ER, Krieger M. Overexpression of the HDL receptor SR-BI alters plasma HDL and bile cholesterol levels. Nature, 1997; 387:414-7.

Kuivenhoven JA, Jukema JW, Zwinderman AH, de Knijff P, McPherson R, Bruschke AV, Lie KI, Kastelein JJ. The role of a common variant of the cholesteryl ester transfer protein gene in the progression of coronary atherosclerosis. The Regression Growth Evaluation Statin Study Group. N Engl J Med, 1998; 338:86-93.
Kumari CS, Govindasamy S, Sukumar E. Lipid lowering activity of Eclipta prostrata in experimental hyperlipidemia. J Ethnopharmacol, $2006 ; 105: 332-5$.

Liu Z-Q, Shan H-Y. Cholesterol, not polyunsaturated fatty acids, is target molecule in oxidation induced by reactive oxygen species in membrane of human erythrocytes. Cell Biochem Biophys, 2006; 45:185-93.

Maciejewska D, Ossowski P, Drozd A, Ryterska K, Jamioł-Milc D, Banaszczak M, Kaczorowska M, Sabinicz A, Raszeja-Wyszomirska J, Stachowska E. Metabolites of arachidonic acid and linoleic acid in early stages of non-alcoholic fatty liver disease - a pilot study. Prostaglandins Other Lipid Mediat, 2015; 121:184-9.

Matyash V, Liebisch G, Kurzchalia TV, Shevchenko A, Schwudke D. Lipid extraction by methyl-tert-butyl ether for highthroughput lipidomics. J Lipid Res, 2008; 49:1137-46.

Mroczek A, Kapusta I, Janda B, Janiszowska W. Triterpene saponin content in the roots of red beet (Beta vulgaris L.) cultivars. J Agric Food Chem, 2012; 60:12397-402.

Mushtaq S, Abbasi BH, Uzair B, Abbasi R. Natural products as reservoirs of novel therapeutic agents. EXCLI J, 2018; 17:420-51.

Ney DM, Lasekan JB, Shinnick FL. Soluble oat fiber tends to normalize lipoprotein composition in cholesterol-fed rats. J Nutr, 1988; 118:1455-62.

Porras D, Nistal E. Protective effect of quercetin on highfat diet-induced non-alcoholic fatty liver disease in mice is mediated by modulating intestinal microbiota imbalance and related. Free Radic Biol, 2017; 102:188-202.

Prabu K, Kanchana N, Sadiq AM. Hepatoprotective effect of Eclipta alba on paracetamol induced liver toxicity in rats. J Microbio Biotechnol Res, 2011; 1:75-9.

Puri P, Baillie RA, Wiest MM, Mirshahi F, Choudhury J, Cheung O, Sargeant C, Contos MJ, Sanyal AJ. A lipidomic analysis of nonalcoholic fatty liver disease. Hepatology, 2007; 46:1081-90.

Puri P, Wiest MM, Cheung O, Mirshahi F, Sargeant C, Min HK, Contos MJ, Sterling RK, Fuchs M, Zhou H, Watkins SM. The plasma lipidomic signature of nonalcoholic steatohepatitis. Hepatology, 2009; 50:1827-38.

Rader DJ, Alexander ET, Weibel GL, Billheimer J, Rothblat $\mathrm{GH}$. The role of reverse cholesterol transport in animals and humans and relationship to atherosclerosis. J Lipid Res, 2009; 50:S189-94.

Reeves PG, Nielsen FH, Fahey GCJ. AIN-93 purified diets for laboratory rodents: final report of the American Institute of Nutrition ad hoc writing committee on the reformulation of the AIN-76A rodent diet. 1993; 123:1939-1951.

Reitman S, Frankel S. A colorimetric method for the determination of serum glutamic oxalacetic and glutamic pyruvic transaminases. Am J Clin Pathol, 1957; 28:56-63.

Rodriguez A, Gena P, Mendez-Gimenez L, Rosito A, Valentí V, Rotellar F, Sola I, Moncada R, Silva C, Svelto M, Salvador J. Reduced hepatic aquaporin-9 and glycerol permeability are related to insulin resistance in non-alcoholic fatty liver disease. Int J Obes (Lond), 2014; $38: 1213-20$

Sanyal D, Mukherjee P, Raychaudhuri M, Ghosh S, Mukherjee S, Chowdhury S. Profile of liver enzymes in non-alcoholic fatty liver disease in patients with impaired glucose tolerance and newly detected untreated type 2 diabetes. Indian J Endocrinol Metab, 2015; 19:597-601.

Serviddio G, Bellanti F, Villani R, Tamborra R, Zerbinati C, Blonda M, Ciacciarelli M, Poli G, Vendemiale G, Iuliano L. Effects of dietary fatty acids and cholesterol excess on liver injury: a lipidomic approach. Redox Biol, 2016; 9:296-305.

Singleton VL, Rossi JA. Colorimetry of total phenolics with phosphomolybdic-phosphotungstic acid reagents. Am J Enol Vitic, 1965; 16:144 LP-158.

Smith A, Bruton JW. colour atlas of histological staining techniques. J Clin Pathol, 1978; 31:289. 
Straub BK, Schirmacher P. Pathology and biopsy assessment of non-alcoholic fatty liver disease. Dig Dis, 2010; 28:197-202.

Sun Z-H, Zhang C, Zhang M. A new benzoic acid derivative from Eclipta prostrata. 2010; 8:244-246.

Tammela T, Enholm B, Alitalo K, Paavonen K. The biology of vascular endothelial growth factors. Cardiovasc Res, 2005; 65:550-63.

Targher G, Chonchol M, Zoppini G, Abaterusso C, Bonora E. Risk of chronic kidney disease in patients with non-alcoholic fatty liver disease: is there a link? J Hepatol, 2011; 54:1020-9.

Wolf C, Quinn PJ. Lipidomics: practical aspects and applications. Prog Lipid Res, 2008; 47:15-36.

Wu CH, Lin MC, Wang HC, Yang MY, Jou MJ, Wang CJ. Rutin inhibits oleic acid induced lipid accumulation via reducing lipogenesis and oxidative stress in hepatocarcinoma cells. J Food Sci, $2011 ; 76: 65-72$.

Xia J, Psychogios N, Young N, Wishart DS. MetaboAnalyst: a web server for metabolomic data analysis and interpretation. Nucleic Acids Res, 2009; 37:W652-60.

Yuan JC, Jiang YH, Sheng ZP. Study progress on the chemical constituents of Herba ecliptae. Asia-Pacific Tradit Med, 2009; 5:125-7.
Zehethofer N, Pinto DM. Recent developments in tandem mass spectrometry for lipidomic analysis. Anal Chim Acta, 2008; 627:62-70.

Zhao Y, Peng L, Yang L-C, Xu XD, Li WJ, Luo XM, Jin X. Wedelolactone regulates lipid metabolism and improves hepatic steatosis partly by AMPK activation and up-regulation of expression of PPARalpha/ LPL and LDLR. PLoS One, 2015; 10:e132720.

\section{How to cite this article:}

Helmy AS, Sherif NM, Ghanem HZ, Ibrahim NA, El Gendy AG, Hussein NS, Abdel-Hamid AZ. Targeted metabolomics reveals the therapeutic impact of Eclipta prostrata on dietinduced non-alcoholic fatty liver disease in rats. J Appl Pharm Sci, 2019; 9(S1):077-090. 
Supplementary Table 1. GC-MS assignments of targeted metabolites identified in treated rat serum after silylation.

\begin{tabular}{|c|c|c|c|c|}
\hline Peeks no. & Metabolites & RT & KI & $\mathbf{m} / \mathbf{z}$ \\
\hline S1 & Bis(trimethylsilyl)carbodiimide & 4.248 & 714.8 & 172 \\
\hline S2 & unknown1 & 4.53 & 730.7 & 204 \\
\hline S3 & Tris(trimethylsilyl)borate & 4.649 & 737.4 & 184 \\
\hline S4 & 2-endo-Hydroxy-4-thiahomoadamantane & 4.763 & 740.8 & 247 \\
\hline S5 & Glycerol 1di-TMS & 5.859 & 806.1 & 73 \\
\hline S6 & Lactic acid, di-TMS & 6.015 & 813.5 & 150 \\
\hline S7 & B-Hydroxybutyric acid (tms) & 8.141 & 935 & 160 \\
\hline S8 & Unknown2 & 9.369 & $1,003.3$ & 212 \\
\hline S9 & Unknown3 & 9.464 & $1,006.6$ & 184 \\
\hline S10 & Urea, N,N'-bis(trimethylsilyl)- (CAS & 10.027 & $1,040.4$ & 147 \\
\hline S11 & Benzoic acid trimethylsilyl ester & 10.111 & $1,045.7$ & 179 \\
\hline S12 & L-Leucine, N-(trimethylsilyl)-, trimethylsilyl ester & 10.914 & $1,090.7$ & 189 \\
\hline $\mathrm{S} 13$ & Phosphoric acid, tris-TMS & 11.052 & $1,098.1$ & 301 \\
\hline S14 & Glycerol2-TRI-TMS ETHER & 11.105 & $1,100.2$ & 135 \\
\hline S15 & Unknown4 & 14.884 & $1,313.4$ & 229 \\
\hline S16 & Alpha-Glycerophosphate 4TMS & 22.831 & $1,782.8$ & 317 \\
\hline S17 & Myristic acid, trimethylsilyl ester & 24.077 & $1,832.3$ & 285 \\
\hline S18 & $\beta$-Galactopyranose, pentakis-TMS & 24.698 & $1,867.8$ & 219 \\
\hline S19 & Glucose 5TMS & 27.49 & $2,025.3$ & 117 \\
\hline S20 & Palmitic acid, trimethylsilyl ester & 27.94 & $2,050.8$ & 57 \\
\hline S21 & Linoleic acid trimethylsilyl ester & 30.844 & $2,214.8$ & 337 \\
\hline S22 & Oleic acid, trimethylsilyl ester & 30.928 & $2,220.4$ & 72 \\
\hline S23 & Stearic acid, trimethylsilyl ester & 31.443 & $2,249.3$ & 303 \\
\hline S24 & Arachidonic acid, trimethylsilyl ester & 33.473 & $2,372.5$ & 161 \\
\hline S25 & Myristic acid, 2,3-bis(trimethylsiloxy)propyl ester & 34.132 & $2,413.3$ & 343 \\
\hline S26 & Palmitoleic acid trimethylsilyl ether & 37.174 & $2,609.1$ & 371 \\
\hline S27 & 2-Monostearin trimethylsilyl ether & 39.402 & $2,766.3$ & 129 \\
\hline S28 & Cholesta-3,5-diene & 39.731 & $2,789.6$ & 430 \\
\hline S29 & Bis(trimethylsilyl)monostearin & 39.941 & 2,803 & 128 \\
\hline $\mathrm{S} 30$ & Cholesta-2,4-diene & 40.743 & 2,864 & 81 \\
\hline S31 & Cholesta-4,6-dien-3-ol, (3ß)- & 40.905 & $2,876.2$ & 366 \\
\hline S32 & Cholest-5-en-3-ol (3ß)-, acetate & 41.205 & $2,897.2$ & 213 \\
\hline $\mathrm{S} 33$ & CHOLESTEROL TMS & 44.618 & $3,163.6$ & 366 \\
\hline S34 & Unknown5 & 50.277 & 3,566 & 431 \\
\hline S35 & 1,3-Dipalmitin trimethylsilyl ether & 50.541 & $3,579.6$ & 371 \\
\hline
\end{tabular}
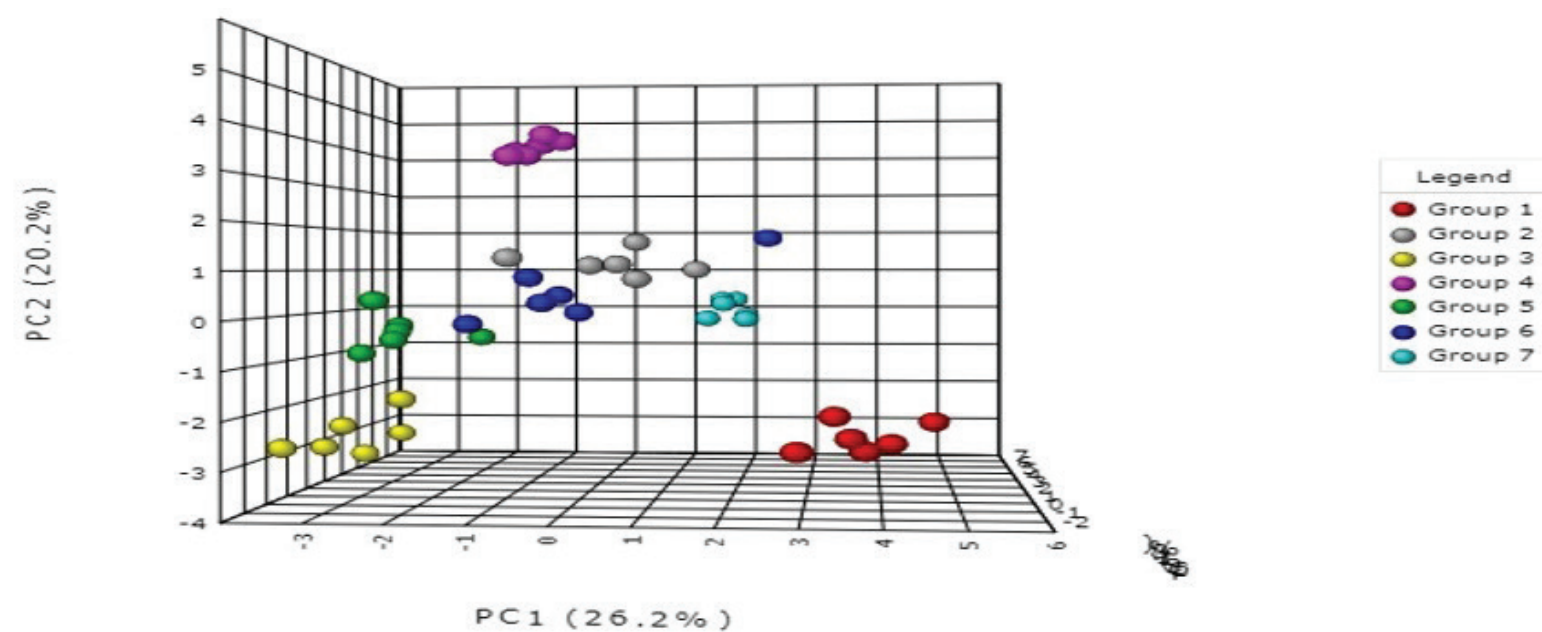

PC $1(26.2 \%)$

Supplementary Figure 1. The PCA scores plots of the seven studied groups derived from GC-MS. The number codes 1, 2, 3, 4, 5, 6, and 7 denote the following different groups: (1) fatty liver group, (2) healthy control, (3) standard drug, (4) extract dose $300 \mathrm{mg} / \mathrm{kg} \mathrm{BW}$, (5) extract dose $200 \mathrm{mg} / \mathrm{kg}$ BW, (6) extract dose $100 \mathrm{mg}$ / $\mathrm{kg} \mathrm{BW}$, and (7) extract dose $50 \mathrm{mg} / \mathrm{kg} \mathrm{BW}$. 
(a)

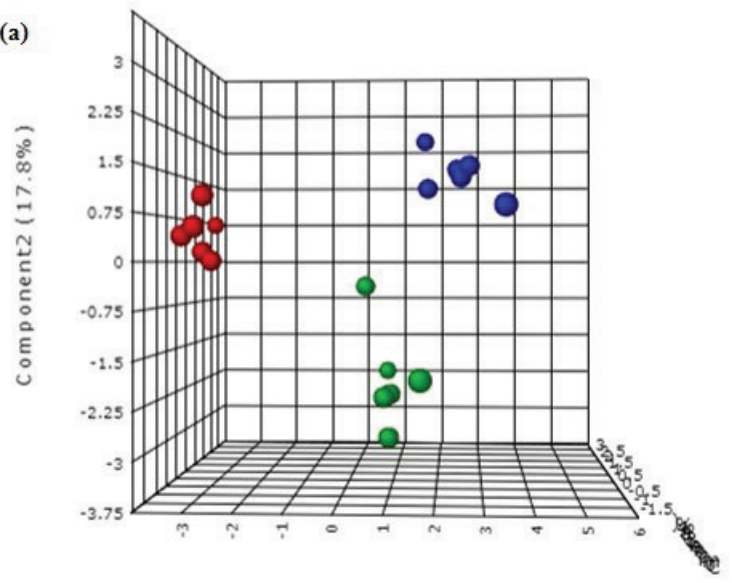

(b)

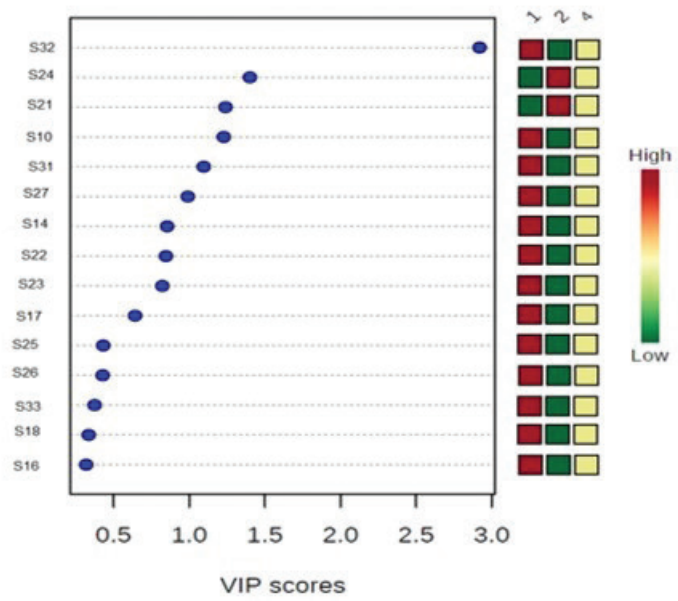

Supplementary Figure 2. PLS-DA score plots obtained from modeling NAFLD (1) healthy control groups 2 and 4 E. prostrata dose (300 mg/kg BW) groups. (a) Score plot of PC1 and PC2. (b) VIP score plot for determination of important metabolites (VIP score > 1). S32: Cholest-5-en-3-ol (3ß)- acetate, S24: Arachidonic acid, S21: Linoleic acid, S31: Cholesta-4,6-dien-3-ol, (3ß)-, S27: 2-monostearin, S14: Glycerol. Peaks follow that listed in Supplementary Table 1 for metabolite identification using GC-MS. The colored boxes on the right indicated the relative concentrations of the corresponding metabolite in each group under study.

(a)

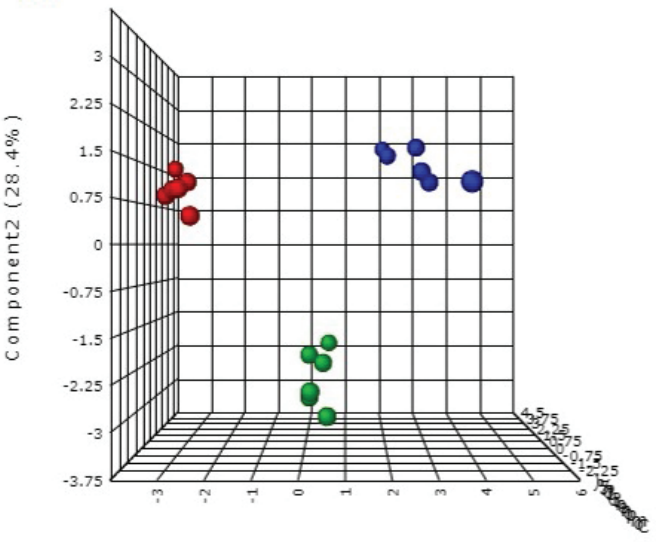

Component1 $(38.7 \%)$ (b)

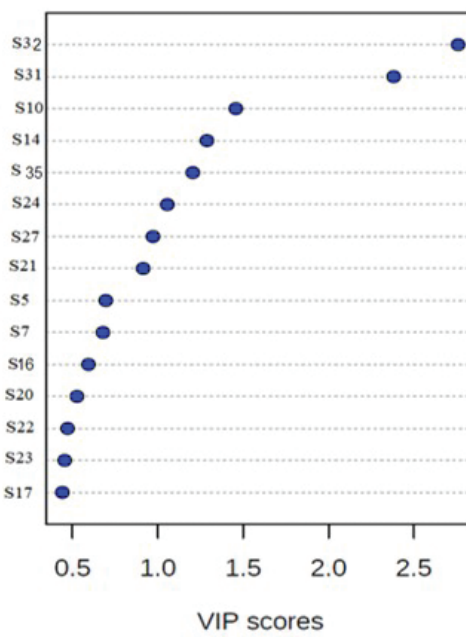

2

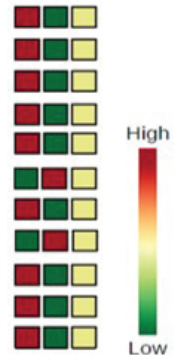

Supplementary Figure 3. PLS-DA score plots obtained from modeling NAFLD (1) healthy control groups 2 and 5 E. prostrata dose (200 mg/kg BW) groups. (a) Score plot of PC1 and PC2. (b) VIP score plot for determination of important metabolites (VIP score > 1). S32: Cholest-5-en-3-ol (3ß)- acetate, S31: Cholesta-4,6-dien3-ol, (3ß)-, S10: Urea, S14: Glycerol, S35: 1,3 dipalmatin, S27: 2-Monostearin, S24: Arachidonic acid, S21: Linoleic acid. Peaks follow that listed in Supplementary Table 1 for metabolite identification using GC-MS. The colored boxes on the right indicated the relative concentrations of the corresponding metabolite in each group under study. 


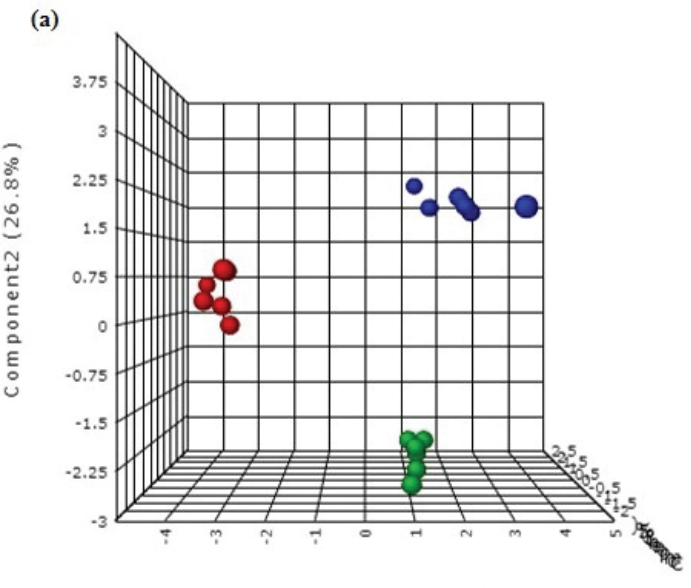

Component1 $(47.4 \%)$ (b)

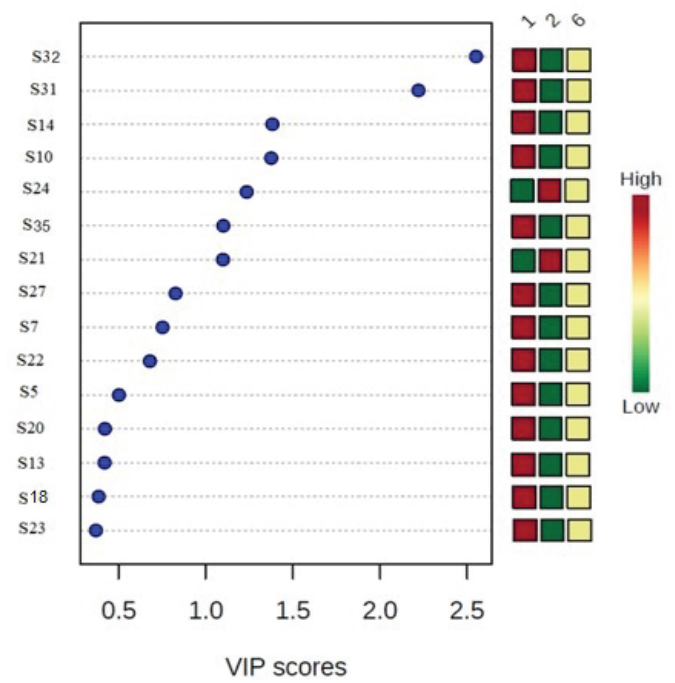

Supplementary Figure 4. PLS-DA score plots obtained from modeling NAFLD (1) healthy control groups 2 and 6 E. prostrata dose (100 mg/kg BW) groups. (a) Score plot of PC1 and PC2. (b) VIP score plot for determination of important metabolites (VIP score > 1). S32: Cholest-5-en-3-ol (3ß)- acetate, S31: Cholesta-4,6-dien3-ol, (3ß)-, S14: Glycerol, S10: Urea, S24: Arachidonic acid, S35: 1,3 dipalmatin, S21: Linoleic acid, S27: 2-Monostearin. Peaks follow that listed in Supplementary Table 1 for metabolite identification using GC-MS. The colored boxes on the right indicated the relative concentrations of the corresponding metabolite in each group under study.

(a)

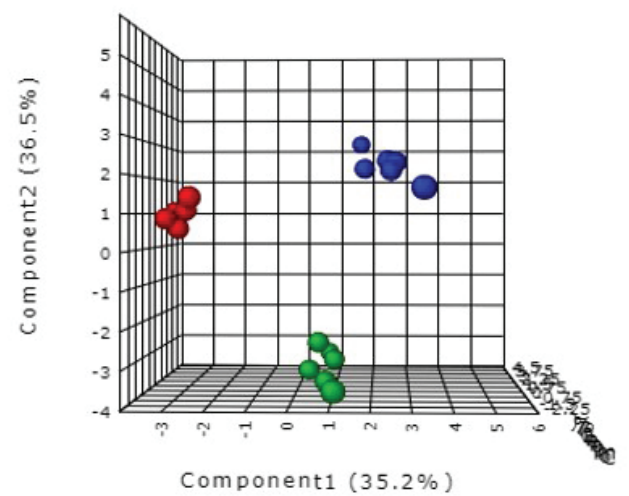

(b)

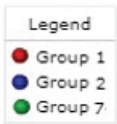

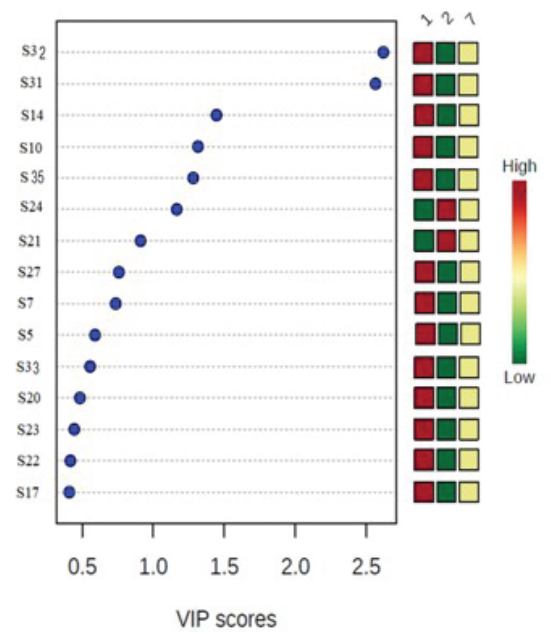

Supplementary Figure 5. PLS-DA score plots obtained from modeling NAFLD (1) healthy control groups 2 and 7 E. prostrata dose (50 mg/kg BW) groups. (a) Score plot of PC1 and PC2. (b) VIP score plot for determination of important metabolites (VIP score > 1). S32: Cholest-5-en-3-ol (3 $\beta$ )- acetate, S31: Cholesta-4,6-dien-3ol, (3ß)-, S14: Glycerol, S10: Urea, S35: 1,3 dipalmatin, S24: Arachidonic acid, S21: Linoleic acid, S27: 2-Monostearin. Peaks follow that listed in Supplementary Table 1 for metabolite identification using GC-MS. The colored boxes on the right indicated the relative concentrations of the corresponding metabolite in each group under study. 